dürfte mit dem Buch jedoch noch nicht gesprochen worden sein.

\section{Matthias Potthoff}

\section{Thorsten Junge}

\section{Jugendmedienschutz und Medienerziehung im digitalen Zeitalter}

Eine explorative Studie zur Rolle der Eltern

Wiesbaden: Springer VS, 2013. - 423 S.

(Medienbildung und Gesellschaft; 24)

ISBN 978-3-658-01536-7

DOI 10.1007/978-3-658-01536-7

(Zugl.: Diss., Fernuniv., Hagen, 2013)

Jugendmedienschutz und Medienerziehung werden gern als Gegensätze gesehen - und verfolgen mit ihren unterschiedlichen Ansprüchen im Spannungsfeld von Bewahren und Befähigen doch ein gemeinsames Ziel: Kinder und Jugendliche beim Aufwachsen in der zunehmend mediatisierten Welt zu unterstützen. Einerseits geht es um einen wirksamen Schutz Heranwachsender vor Medieninhalten, die sie in ihrer Entwicklung beeinträchtigen oder (schwer) gefährden können, anderseits um die Förderung eines selbstbestimmten, reflexiven und partizipativen Medienhandelns. Neben dem Staat, den Medienanbietern und den Institutionen von Bildung und Erziehung kommt hier den Eltern eine besondere Rolle zu, und dieser nähert sich der Autor der zuvor als Dissertation an der Fernuniversität Hagen eingereichten Schrift mit einer explorativen Studie an.

Nach einführenden Anmerkungen zum Aufwachsen im digitalen Zeitalter referiert der $\mathrm{Au}$ tor etwas unvermittelt theoretische Grundlagen zur Wirkung von Medien, ohne dass hier wirklich Neues zu Tage befördert und ein besonderer Nutzen für die eigene Arbeit deutlich wird. Der sich anschließende Teil zum aktuellen Diskurs des Gefahrenpotenzials digitaler Medien bringt einen dann schon deutlich näher an das eigentliche Thema heran und stellt das breite Spektrum an Risiken für die verschiedenen Bereiche digitaler Medien differenziert dar. Dabei werden aktuelle Entwicklungen zielsicher aufgenommen und neben problematischen Medieninhalten (z. B. Gewalt, Pornografie, Radikalismus) auch prekäre Umgangsformen (z. B. Cyber-Mobbing, Umgang mit privaten Daten und Persönlichkeitsrechten) in den Blick genommen.

Im nachfolgenden Teil zum Jugendmedienschutz in Deutschland sind zwar nicht alle restriktiven Maßnahmen, die bei digitalen Medien eine Rolle spielen, systematisiert nachzulesen, der Autor entfaltet aber abschließend zentrale Aspekte (z. B. Anpassung rechtlicher Grundlagen, Internationalisierung, Transparenz), die den Diskurs zu den Perspektiven des Jugendmedienschutzes im digitalen Zeitalter bereichern. Der hierauf folgende Teil zur Medienerziehung bildet mit seiner Fokussierung auf Medienkompetenzvermittlung das Ziel nur unvollständig ab, weil Selbstlern- und Selbstsozialisationsprozesse auch im Rahmen institutionalisierter Medienkompetenzförderung immer wichtiger werden. Der längere Abschnitt zur Medienerziehung der Eltern ist demgegenüber mit seiner differenzierten Beschreibung relevanter Einflussfaktoren mehr als nur lesenswert.

Der besondere Wert des Buches liegt zweifelsohne in den Ergebnissen der explorativen Studie. Mit teilstrukturierten Paarinterviews als methodischem Zugang sollte die familiäre Wirklichkeit nicht nur verbalisiert, sondern auch in der Befragungssituation sichtbar werden. Diese besondere Form der qualitativen Datenerhebung ist mit Blick auf das Erkenntnisinteresse durchaus sinnvoll, aber mit dem Problem verbunden, dass Eltern in einer solchen Befragungssituation tendenziell bestrebt sind, hier ihre Differenzen nicht unbedingt zu offenbaren. Auch familiäre Medienerziehung "lebt" aber von unterschiedlichen Sichtweisen der Erziehenden, die in ihrer Umsetzung dem oder der anderen nicht unbedingt zur Kenntnis gelangen (sollen).

Die Befragung von letztlich insgesamt 14 Elternpaaren erscheint auf den ersten Blick etwas reduziert, die Analyse des umfangreichen erhobenen Materials in der Tiefe und unter Einbezug wichtiger Kontexte wie Medienausstattung und Mediennutzung, Themen und Kontroversen in den Familien erlaubt aber einen sehr differenzierten Einblick in das praktizierte medienerzieherische Handeln, in zugrunde liegende Leitlinien, übergeordnete Erziehungskonzepte und in die auf bestimmte (Gefahren-)Bereiche der Mediennutzung Heranwachsender fokussierte Wahrnehmung von Eltern.

In der sehr gelungenen, ausführlichen Darstellung der Ergebnisse wird zunächst entlang wesentlicher Punkte (Stellenwert im Familienalltag, Kommunikation und Kontroversen etc.) die Rolle der Medien in den Familien herausgearbeitet. Anschließend geht es um das konkrete medienerzieherische Handeln der Eltern, welches in der Gesamtbetrachtung offenbar fast ausschließlich an den Risiken und kaum an den Chancen digitaler Medien für Heranwachsende orientiert ist. Differenziert nach Internetnut- 
zung, Online-Kommunikation, Computerspiel- und Mobiltelefonnutzung erhält man hier wertvolle Einblicke in die angewandten Regeln und Vorgaben. Zentrale Aspekte werden mit Interviewzitaten veranschaulicht, und auch Reflexionen der Eltern zur Wirksamkeit haben ihren Platz.

Bemerkenswert ist, wie der Autor mit der Methode des axialen Kodierens den Hintergrund des medienerzieherischen Elternhandelns hinsichtlich ursächlicher und intervenierender Bedingungen, zentraler Kontexte, Handlungsstrategien und Konsequenzen im Zusammenhang darstellt und mit einem Fallbeispiel (Anschaffung des Computerspiels Call of Duty) plastisch macht. Die in der abschließenden Typologisierung ordentlich herausgearbeiteten Typen eines „Freiraum gewährenden“, „begrenzenden“, „regulierend-kontrollierenden “, „,autoritär-bewahrenden “ und „unsicheren" medienerzieherischen Elternhandelns veranschaulichen eindrucksvoll eine Diversität, die - wie im Resümee völlig zu Recht hervorgehoben - auch bei der adäquaten Zielgruppenansprache medienpädagogischer Arbeit mit Eltern Beachtung finden sollte.

Alles in allem bietet das Buch differenziert Einblick in die praktizierte, v. a. an den Gefahren digitaler Medien orientierte Medienerziehung in Familien. Besonders wertvoll sind die erzielten Ergebnisse für die Arbeit von Multiplikatoren, die Eltern für den Medienumgang ihrer Kinder sensibilisieren und ihnen konkrete Handlungsalternativen aufzeigen wollen. Eltern selbst bieten sie die Möglichkeit, das eigene Handeln zu reflektieren und es mit Kenntnis wirksamer Konzepte (anderer) an den Erfordernissen in der eigenen Familie auszurichten. Medienpolitiker und Jugendmedienschützer erhalten wichtige Hintergründe zu den Orten, die noch immer ein zentrales Moment beim besonderen Schutzbedürfnis und Medienkompetenzerwerb Heranwachsender sind: die Familien.

Daniel Hajok

\section{Benjamin Krämer \\ Mediensozialisation}

Theorie und Empirie zum Erwerb medienbezogener Dispositionen

Wiesbaden: Springer VS, 2013. - 509 S.

ISBN 978-3-531-19567-4

(Zugl.: München, Univ., Diss., 2012)

„Warum nutzen wir die Medien so, wie wir es tun?" Mit dieser deutlich von der Mediennut- zungsforschung geprägten Frage steigt Benjamin Krämer in seine Dissertation zur Mediensozialisation ein. Er setzt sich darin zum einen das Ziel, eine breite theoretische Basis zur Mediensozialisation im Allgemeinen zu schaffen und zum anderen diese Theorie mit Hilfe einer quantitativen Befragung zu prüfen. Dabei schränkt er jedoch bereits zu Beginn ein, dass seine Erhebung „nur eine erste selektive Prüfung der theoretischen Ansätze erlauben“" (S. 17) wird. Krämer fragt nach der Mediennutzung und den Genrevorlieben in Abhängigkeit der jeweiligen lebensweltlichen Hintergründe (soziale Klasse, Kapitalien etc.) einer Person und ihres Elternhauses. Mediensozialisation definiert er für seine Arbeit als „Erwerb von Dispositionen“ (S. 428, ausführlich S. 32).

Krämer grenzt sich in seinem Verständnis von einer stärker medienpädagogisch orientierten Perspektive ab, die eher danach fragt, welche Rolle Medien in der Entwicklung von Kindern und Jugendlichen spielen. Insofern werden die dort erzielten Erkenntnisse über die Rolle der Medien in der Sozialisation auch nicht oder nur kaum thematisiert. Der Autor verfolgt dabei die Prämisse, medienbezogene Dispositionen vor allem mit Bezug zur Sozialstruktur $\mathrm{zu}$ untersuchen, also welche Rolle „stratifikatorische Differenzierung oder soziale Ungleichheit" (S. 36, H.i.O.) spielen. Für die Sozialisation ebenfalls relevante sozio-emotionale Faktoren in der Familie werden im Forschungsdesign von Benjamin Krämer dagegen nicht aufgegriffen. In seiner Befragung beschränkt er sich zudem aus Gründen der Handhabbarkeit auf die Genrevorlieben und Rezeption von Musik und Fernsehen.

Der Band ist logisch und gut nachvollziehbar aufgebaut. Im Theorieteil bespricht Krämer umfassend Begriffe und theoretische Konzepte wie (Medien-)Sozialisation, Sozialstruktur, Dispositionen und ihren Erwerb, unterschiedliche Sozialisationsinstanzen, Mediensozialisation im Zeitverlauf sowie die Rolle von Identität, Biographie, Generationen und sozialen Bewegungen. Insbesondere bedient er sich bei den Konzepten von Feld, Kapital und Habitus aus den Arbeiten von Pierre Bourdieu. Dabei bespricht er die wesentlichen Theoriestränge der (Medien-)Sozialisationsforschung und arbeitet die relevante Literatur überzeugend auf. Einige grundlegende Autoren der Mediensozialisationsforschung wie Johannes Fromme oder Dieter Baacke werden jedoch außer Acht gelassen. Krämer legt gleich zu Beginn eine präzise Charakterisierung des Forschungsfeldes der Mediensozialisation vor, die er von der Medienwirkungsforschung abgrenzt. Bedauernswert ist 